\title{
Artefatos de auxílio de desenvolvimento de Recursos Educacionais Abertos (REA) com foco na colaboração
}

\author{
Open Educational Resources (OER) development assistance artifacts \\ with a focus on collaboration
}

\author{
Gabrielle da Fonseca Hartmann Grimm, Adriano Heemann
}

design colaborativo, recursos educacionais abertos, artefatos, modelo

O presente artigo apresenta um estudo que objetiva analisar os artefatos existentes para auxílio ao desenvolvimento de Recursos Educacionais Abertos (REA). O enfoque neste tema tem como antecedente a aguda problemática enfrentada pelo setor da educação no Brasil, que aponta para uma demanda crescente por artefatos de suporte a um tipo novo de ensino-aprendizagem, cada vez mais aberto e colaborativo. O método deste estudo é a revisão bibliográfica, iniciada de forma narrativa (RBN) e aprofundada de maneira sistemática (RBS). A partir da RBN e RBS foram selecionados artefatos para auxílio no desenvolvimento de REA para uma análise paramétrica. Os resultados do estudo apontam para a existência de oito artefatos que enfatizam a importância da colaboração no ciclo de vida de REA. Porém, seis deles apontam no artefato a colaboração como algo que acontece intuitivamente, como fundamental, porém sem abordar uma estratégia para isso, dois deles apresentam algumas diretrizes para a colaboração e seus artefatos são descritos no artigo. Como conclusão, o estudo sugere que os artefatos existentes são restritos à importância do desenvolvimento colaborativo porém não consideram a colaboração de maneira processual, porém ainda é necessário aprofundar como os participantes de um projeto podem conduzir as etapas de um REA de maneira colaborativa.

collaborative design, open educational resources, artifacts, model

This paper presents a study that aims to analyze the existing artifacts to assist the development of Open Educational Resources (OER). The focus on this theme has as an antecedent the acute problem faced by the education sector in Brazil, which points to a growing demand for artifacts to support a new type of teaching-learning, increasingly open and collaborative. The method of this study is the literature review, started in a narrative way (RBN) and deepened in a systematic way (RBS). From the BNR and SBR, artifacts to aid in the development of OER were selected for a parametric analysis. The results of the study point to the existence of eight artifacts that emphasize the importance of collaboration in the OER life cycle. However, six of them point out in the artifact collaboration as something that happens intuitively, as fundamental, but without addressing a strategy for it, two of them present some guidelines for collaboration and their artifacts are described in the article. In conclusion, the study suggests that the existing artifacts are restricted to the importance of collaborative development but do not consider collaboration in a procedural way, but it is still necessary to deepen how the participants of a project can conduct the stages of an OER in a collaborative way.

Anais do $10^{\circ} \mathrm{CIDI}$ e $10^{\circ} \mathrm{CONGIC}$

Kelli C.A.S. Smythe, Rafael de Castro Andrade (orgs.)

Sociedade Brasileira de Design da Informação - SBDI

Curitiba | Brasil | 2021
Proceedings of the $10^{\text {th }} \mathrm{CIDI}$ and $10^{\text {th }}$ CONGIC

Kelli C.A.S. Smythe, Rafael de Castro Andrade (orgs.)

Sociedade Brasileira de Design da Informação - SBDI Curitiba | Brazil | 2021 


\section{Introdução}

O desenvolvimento tecnológico vem modificando o acesso à informação, as atividades cotidianas bem como a área da educação de um modo geral. Nessa área, nos últimos anos diversos projetos e tecnologias vêm sendo elaborados e utilizados de modo a amplificar o processo de ensino-aprendizagem. Mas para que novos recursos tecnológicos possam impactar positivamente também no desenvolvimento social e na disseminação do conhecimento é necessário um tipo de interação colaborativa em rede e orientado à educação. Uma iniciativa que permite ampliar a disponibilidade de recursos educacionais é o movimento openness. Dentro desse movimento, os Recursos Educacionais Abertos (REAs) disponibilizam materiais educacionais digitais de forma livre e aberta ao ensino, aprendizagem e pesquisa, e visam a colaboração entre professores e estudantes e o compartilhamento de materiais abertos para tornar a educação mais acessível, democrática e eficaz. Recursos educacionais são materiais ou artefatos, que são desenvolvidos e utilizados nos momentos de ensinoaprendizagem, e podem ser nos formatos digital ou impresso. Para que um recurso seja considerado educacional é necessário "que ele se torne condicionante e estruturador do processo de ensino-aprendizagem" (Mallmann \& Nobre, 2015, p.623). Um recurso educacional é então um recurso, material, artefato que foi planejado e organizado com intencionalidade pedagógica para aquele momento. Segundo Mallmann e Nobre (2015) a mediação proporcionada por um recurso educacional (ou recurso didático) têm dois principais aspectos: na abordagem dos conceitos ou nas situações que desafiam à aprendizagem (Mallmann \& Nobre, 2015). O conceito de REA é focado em dois princípios: licenças que permitam uma flexibilidade e uso legal dos recursos didáticos; e abertura técnica, com uso de formatos que permitam a adaptação e redistribuição.

Com seu potencial de desenvolvimento coletivo, REA deve ser elaborado unindo e integrando habilidades. Se um grupo desenvolve um recurso e não possui conhecimento sobre algum aspecto, outro grupo pode modificar e acrescentar conforme suas habilidades. Assim os recursos tendem a melhorar sua qualidade em cada versão modificada. A colaboração em um projeto REA pode acontecer de várias maneiras: (a) de forma síncrona, com uma equipe multidisciplinar, com participantes de diversas áreas e habilidades, assim como os usuários finais, que são os estudantes e educadores; (b) e de forma assíncrona, com participantes que contribuem e melhoram a qualidade de determinado REA que já foi desenvolvido por outros autores, também com participação ativa de estudantes e educadores. A participação ativa dos estudantes na construção de recursos educacionais é uma característica importante na Educação Aberta.

Nesse sentido, a colaboração entre os atores envolvidos no desenvolvimento de REAs é fundamental, segundo Okada (2013) quanto mais autores usarem e partilharem REA, mais coautores podem contribuir para o fluxo REA, o fluxo acontece em movimento espiral que aumenta a reutilização, bem como a re-autoria, coautoria e coaprendizagem. Porém, muitos desconhecem como isso pode ser realizado, tanto no sentido das delimitações de REA, tanto em como organizar um trabalho coletivo e colaborativo para estimular a disseminação de conhecimento por meio da educação aberta. 
Neste contexto, o presente artigo apresenta um estudo que objetiva analisar os artefatos existentes para auxílio ao desenvolvimento de Recursos Educacionais Abertos (REA), e identificar se e como a colaboração é abordada nos mesmos.

\section{Colaboração e Design}

A colaboração é um substantivo feminino e usado nas práticas cotidianas, para se referir ao ato de se trabalhar ou elaborar algo em conjunto com outros. Segundo Hargrove (1998, p.25) a colaboração "implica em fazer alguma coisa junto com outro e esse é exatamente seu significado". O fazer alguma coisa junto com o outro é utilizado para atingir objetivos e resolver problemas com melhores resultados do que se fosse individualmente. Ainda segundo o autor, as pessoas que colaboram são aquelas capazes de reconhecer que somente suas experiências, seu potencial, não são suficientes para tornar algo realidade, elas necessitam buscar outros talentos e habilidades para unir forças (Hargrove, 1998).

Para isso, muitas vezes é necessário mudar o comportamento e criar novas formas de pensar em soluções e de como trabalhar em conjunto. Situações e problemas complexos exigem uma rede de conhecimentos e habilidades que não é possível por meio do indivíduo isolado. Segundo Hargrove (1998) as pessoas se inspiram a colaborar quando têm um objetivo complexo e que é muito significativo para si, e reconhecem que não conseguem realizá-lo sozinhas. A busca por ideias e soluções se torna mais dinâmica e inovadora ao participar de uma diversidade de perspectivas e experiências. Nesse sentido, é necessária uma postura de aprendizado constante e abertura para ideias e percepções diferentes.

Segundo Hargrove (1998) a colaboração não é algo que acontece por acidente, sem esforço, ela ocorre propositalmente, e não envolve somente questões técnicas mas também de atitude, "as pessoas colaboram com maior eficiência quando têm os instrumentos e métodos adequados para ajudá-las a reforçar uma nova disposição mental" (Hargrove, 1998, p.86). Uma nova disposição mental deve ser exercitada, com mudanças no pensamento e nas atitudes, muitas vezes é preciso aprender como realizar colaborações, principalmente na esfera do trabalho, para romper velhos padrões de competição e especialização individual.

A colaboração não acontece por acaso, como já citado anteriormente, não acontece intuitivamente, é preciso esforço, disposição e coordenação, e depende de habilidades, atitudes e comportamentos de pessoas, que são diferentes umas das outras. Com isso, Hargrove (1998) reforça que mesmo com planejamento, não existe uma receita específica pronta para colaboração. Existe reflexão, princípios e elementos para se atingir melhores resultados. O objetivo da colaboração não é a colaboração por si só, a colaboração é um meio para se atingir uma finalidade, e a finalidade são melhores resultados (Hansen, 2010). Dessa forma, ela deve ser pensada estrategicamente, por isso Hansen (2010) denomina um conjunto de princípios como colaboração disciplinada, pois atingir os objetivos esperados fica mais palpável se a pessoa sabe como colaborar. 
Para Hansen (2010) a colaboração disciplinada contém três passos:

I. Avaliar sobre a necessidade da colaboração: A colaboração é algo que demanda tempo, esforços e recursos e deve-se avaliar se será benéfico realmente. Segundo Hansen (2010) é necessário avaliar a vantagem e desvantagem, pois em alguns casos, é melhor não colaborar, se não existir uma razão para tal, "ser disciplinado sobre colaboração é saber quando não colaborar" (Hansen, 2010, p. 17). Deve-se avaliar quais são as metas e os objetivos, e se melhores resultados serão obtidos, inovações melhores, mais vendas ou melhores custos, ou operações mais eficientes a partir de um trabalho colaborativo. Insistir na colaboração quando não necessário, pode impactar negativamente um projeto, como demandar mais tempo, mais recursos e desgaste das pessoas envolvidas. Ao avaliar as oportunidades, pode-se identificar que a colaboração será necessária somente em determinadas etapas ou tarefas.

II. Se existem barreiras para a colaboração: A colaboração envolve comportamento e atitudes, e nem sempre as pessoas conseguem colaborar, e são várias as barreiras que podem impedir que as pessoas explorem o potencial da colaboração. É importante identificar quais são as barreiras para definir soluções para essas barreiras. Hansen (2010) aponta quatro principais barreiras: barreira do "não inventado aqui", barreira de retenção de informações, barreira na busca de informações e barreira de transferência. Situações diferentes têm barreiras diferentes, e identificar as barreiras contribuem para que se elaborem soluções (Hansen, 2010).

III. Conceber soluções para eliminar as barreiras: assim que identificadas as barreiras é possível elaborar soluções para as mesmas (Hansen, 2010). As barreiras do "não inventado aqui" e de retenção de informações são barreiras motivacionais e exigem que sejam utilizados mecanismos para motivar as pessoas que precisam colaborar, já as barreiras de busca de informações e de transferência são barreiras de habilidade, são necessários mecanismos para ensinar novas habilidades para que as pessoas possam colaborar. Hansen (2010) lista três mecanismos que líderes ou gestores podem combinar para eliminar as barreiras à colaboração: mecanismo de unificação, mecanismo de pessoas e mecanismo de redes.

Com isso, é necessário esforço e planejamento para o trabalho colaborativo, e os participantes devem ter um entendimento e uma visão compartilhada do que estão criando e sobre todo o processo.

\section{Metodologia}

Com o intuito de identificar projetos de Recursos Educacionais Abertos que consideram processos de design e colaboração e analisar os artefatos utilizados para apoiar esses projetos, foram realizados procedimentos como revisão bibliográfica narrativa e sistemática (RBN e RBS) e posteriormente, com os projetos identificados foi realizada análise paramétrica. 
Figura 1: Procedimentos adotados

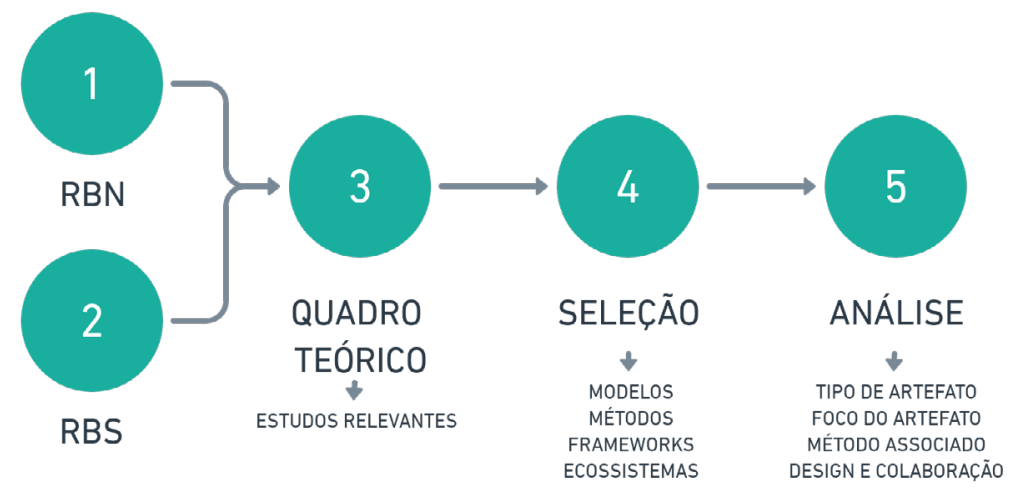

A partir dos dados coletados, tanto na RBS quanto na RBN são elaborados quadros teóricos de Colaboração e Design Colaborativo e de Recursos Educacionais Abertos. Com o quadro teórico é possível identificar dentre as publicações, quais delas são selecionadas para compor os artefatos similares para a análise paramétrica, e quais delas apresentam constructos teóricos. As publicações aptas para análise são as que apresentam como foco: modelos, métodos, frameworks e ecossistema.

\section{Revisão bibliográfica sistemática e narrativa}

A revisão bibliográfica é importante para reconhecer as bases teóricas que irão fundamentar a pesquisa, assim como identificar os estudos já realizados sobre a temática investigada, por meio de livros, teses, dissertações e artigos científicos (Gil, 2008; Marconi \& Lakatos, 2003).

A pesquisa foi iniciada com uma revisão bibliográfica narrativa (RBN) para conhecer os principais conceitos e termos sobre o desenvolvimento de recursos educacionais abertos e sobre o design colaborativo. A partir da RBN pode-se elaborar o protocolo da revisão bibliográfica sistemática (RBS), visto que a RBS é um instrumento para mapear trabalhos publicados no tema de pesquisa, com rigor científico, justificável, que possibilita a identificação de ênfases e lacunas na área investigada (Conforto et al., 2011).

A RBS foi conduzida com base no roteiro proposto por Conforto, Amaral e da Silva (2011), que dividem a revisão em três fases: entrada, processamento e saída. A partir do objetivo do estudo foram delimitadas as fontes primárias para revisão nas bases de dados de artigos científicos: periódicos da Capes, Scopus e Web of Science. As pesquisas foram delimitadas com os seguintes critérios de inclusão e qualificação: artigos publicados nos últimos 5 anos (2015-2019), artigos revisados por pares, em inglês ou português.

Com base na RBN foi possível determinar as palavras ou termos para compor as strings de busca nas bases de dados. Nas discussões que cercam a colaboração, aparecem termos como (português): aprendizagem colaborativa, colaboração interinstitucional, coletividade aberta de pesquisa, cultura participativa, produção coletiva, codesign de REA, co-criação, tecnologias ou ferramentas de colaboração. Nos termos que envolvem os recursos educacionais abertos aparecem termos como (português): aprendizagem aberta, educação aberta, práticas e políticas educacionais abertas. Com isso, foram obtidos dados brutos, que 
passaram por um filtro preliminar (FP), o próximo passo foi o filtro 1 "F1", na sequência o filtro 2 "F2" e o filtro 3 "F3". Com base na leitura dos artigos selecionados, foram delimitados tópicos relevantes para o estudo, para que fosse possível avaliar os artigos. Os tópicos definidos consideram se o artigo aborda: a construção coletiva do conhecimento; o desenvolvimento REA colaborativo de maneira intuitiva; ou o desenvolvimento REA colaborativo de maneira sistematizada e planejada; ressalta a importância da colaboração na produção de REA; o uso de ferramentas de autoria e cocriação; descreve algum modelo de design ou redesign de REA; descreve metodologias de design participativo na criação de REA. Os últimos dois tópicos visam demarcar se o artigo era um relato de experiência, revisão de literatura ou modelo teórico.

Foram destacados estudos que desenvolveram artefatos para ampliar o alcance de REA, sejam modelos, frameworks, métodos ou relatos de projeto.

\section{Análise paramétrica de similares}

Com os artefatos selecionados, o procedimento a ser realizado foi a análise paramétrica, para identificar e descrever os elementos destacados nos artefatos, assim como realizar uma comparação entre eles por meio de parâmetros estabelecidos com base na classe de problemas do pretendido modelo (Pazmino, 2009). A análise dos artefatos, é apresentada primeiro em síntese em um quadro, e posteriormente contempla uma descrição de cada artefato selecionado. Os parâmetros da análise são:

I. Tipo de artefato: esse parâmetro visa identificar quais artefatos outras pesquisas têm desenvolvido, e delimitar qual o tipo de artefato está descrito, se é um modelo, um método, são recomendações ou diretrizes.

II. Foco do artefato: esse parâmetro visa delimitar se o foco do artefato está nos aspectos jurídicos, sociais ou técnicos de projetos REA.

III. Método de desenvolvimento associado: esse parâmetro visa observar se no artefato proposto existe algum outro método ou processo associado ao fluxo de desenvolvimento de REA, exemplo: Design instrucional, Metodologias ágeis, Design Thinking, entre outros.

IV. Design e colaboração: esse parâmetro visa observar se o artefato aborda as questões sobre design e sobre colaboração, a colaboração é intuitiva ou disciplinada.

\section{Resultados e Discussão}

Os resultados obtidos a partir de 16 artigos selecionados, evidenciam a relevância da colaboração para a educação aberta (e aprendizagem aberta) e como ponto fundamental para o ciclo de vida REA. Os artigos abordaram projetos desde design de REA, mapeamento de comportamento colaborativo, aprendizagem colaborativa e desenvolvimento de artefatos (modelos, métodos, frameworks e ecossistemas) para impulsionar os REAs.

Grande parte dos artigos (12 artigos) propõe o uso de ferramentas de autoria e cocriação para incentivar a colaboração entre as partes envolvidas em um projeto REA, algumas ferramentas 
foram desenvolvidas especificamente para esse propósito, e outras possibilitam a colaboração por suas características da web 2.0. A colaboração é ressaltada como essencial, são sugeridas ferramentas para facilitação, mas a colaboração é tratada como uma ação que acontece de maneira intuitiva, que naturalmente ocorre pela ação de contribuir, somar, acrescentar.

Alguns artigos (7 artigos) descrevem processos de design de REA ou metodologias de desenvolvimento para permitir a reutilização, ou redesign, assim como ferramentas do design participativo para elaboração de recursos com participação ativa dos usuários na construção. E somente um artigo aborda a colaboração de maneira sistematizada, porém o foco do estudo de Pirkkalainen et al (2016) é comportamental, aborda como estimular a colaboração como comportamento em práticas educacionais colaborativas, pensando nos estudantes como alvo.

Todos os artigos selecionados na RBS, assim como alguns selecionados a partir da RBN, foram organizados em uma tabela com os dados do artigo, como: autores, ano, título, fonte de coleta (se RBS ou RBN), qual o foco do artigo (se aborda conceitos, apresenta método, modelo ou melhores práticas). Os artigos que tem por foco métodos, modelos ou frameworks foram selecionados para análise paramétrica. A tabela a seguir apresenta os artefatos selecionados e analisados, assim como uma síntese dos parâmetros analisados.

Tabela 1: Artefatos selecionados para análise

\begin{tabular}{|c|c|c|c|c|c|c|}
\hline Coleta & Autor/es & Ano & $\begin{array}{l}\text { Tipo de } \\
\text { artefato }\end{array}$ & Foco & Método Associado & $\begin{array}{l}\text { Design e } \\
\text { colaboração }\end{array}$ \\
\hline RBN & Santana & 2019 & Metamodelo & Técnico & Design Instrucional & $\begin{array}{l}\text { Aponta algumas } \\
\text { diretrizes de } \\
\text { colaboração }\end{array}$ \\
\hline RBS & Kim & 2018 & Framework & Técnico & $\begin{array}{l}\text { Teoria da cognição } \\
\text { distribuída e } \\
\text { aprendizagem } \\
\text { baseada em } \\
\text { exemplos }\end{array}$ & $\begin{array}{l}\text { Colaboração } \\
\text { intuitiva }\end{array}$ \\
\hline RBN & Teodoroski & 2018 & Ecossistema & Social & Nenhum & $\begin{array}{l}\text { Colaboração } \\
\text { intuitiva }\end{array}$ \\
\hline RBS & Leffa & 2017 & $\begin{array}{l}\text { Práticas } \\
\text { abertas }\end{array}$ & Social & Nenhum & $\begin{array}{l}\text { Colaboração } \\
\text { intuitiva }\end{array}$ \\
\hline RBS & Arimoto et al & 2016 & Método & Técnico & SCRUM e XP & $\begin{array}{l}\text { Colaboração } \\
\text { disciplinada }\end{array}$ \\
\hline RBS & $\begin{array}{l}\text { Nascimbeni } \\
\text { e Burgos }\end{array}$ & 2016 & Estrutura & Social & Nenhum & $\begin{array}{l}\text { Colaboração } \\
\text { intuitiva }\end{array}$ \\
\hline RBS & Ávila et al & 2016 & Modelo & Técnico & $\begin{array}{l}\text { Design instrucional, } \\
\text { Design Universal } \\
\text { para Aprendizagem }\end{array}$ & $\begin{array}{l}\text { Colaboração } \\
\text { intuitiva }\end{array}$ \\
\hline RBN & Zancanaro & 2015 & Framework & Técnico & $\begin{array}{l}\text { Design instrucional } \\
\text { e Engenharia do } \\
\text { conhecimento }\end{array}$ & \\
\hline
\end{tabular}

Dos artefatos selecionados (8 artefatos), todos mencionam a relevância no desenvolvimento colaborativo dos REA e a importância da colaboração para a educação aberta. Na grande maioria (6 artigos) colaboração é ressaltada como essencial, são sugeridas ferramentas para 
permitir, mas é tratada de maneira intuitiva, que acontece naturalmente pela ação de contribuir, somar, acrescentar (Kim, 2018; Teodoroski, 2018; Leffa, 2017; Nascimbeni e Burgos, 2016; Ávila et al, 2016; Zancanaro, 2015). Os oito artefatos foram analisados e descritos, porém serão detalhados neste artigo os dois que se mostraram mais relevantes para o estudo. Os dois artefatos detalham estratégias ou diretrizes para o desenvolvimento colaborativo, e são o metamodelo de Santana (2019) e o método de Arimoto et al (2016), e serão abordados a seguir.

\section{Artefato de Santana (2019)}

Santana (2019) desenvolveu um metamodelo para o design de REA com a preocupação com o contexto cultural, com guias para serem analisadas durante o design de um REA multicultural e que possam auxiliar na criação de artefatos de um REA ou um REA inteiro.

O metamodelo elaborado foi chamado de REAM (Recurso Educacional Aberto Multicultural) e é dividido em quatro componentes: RMGuidelines, RMQuestionnaire, iREAM, iREAMTools. O metamodelo é constituído de cinco dimensões (Santana, 2019):

- Planejamento - realização de atividades para delimitar a demanda educacional que requisita um REA (contexto cultural, público-alvo, definir objetivos e abordagens do REA, etc);

- Colaboração - efetivação de atividades que buscam estruturar, regular e incentivar a participação no processo de design, e também o trabalho colaborativo entre os participantes;

- Licenciamento - descrição dos termos de licença associados ao REA produzido, envolvendo as partes responsáveis pela autoria do REA;

- Avaliação - realização de testes; avaliação de questões de aceitabilidade, acessibilidade e usabilidade; e qualidade do recurso;

- Produção - implementação do projeto, produção dos conteúdos e desenvolvimento dos artefatos de softwares necessários, tendo como base os princípios $5 R$ s para promover o reuso em seus diversos níveis de REAs já existentes.

Cada dimensão possui um conjunto de diretrizes que auxiliam o designer e a equipe. Santana reforça que o metamodelo não fornece um processo para o desenvolvimento, mas um modelo genérico que pode ser associado a outros modelos, como por exemplo o modelo ADDIE (de design instrucional). Na dimensão colaboração, por exemplo, são seis diretrizes que têm por foco os grupos de colaboração na produção de REA. As diretrizes para a colaboração são:

- Incluir um ou mais representantes dos futuros usuários do REA;

- Criar um ambiente que possibilite e incentive o usuário a se engajar ativamente no design do REA;

- Dar voz às opiniões de todos os envolvidos durante o design de REA;

- Respeitar as opiniões e as ideias dos participantes, principalmente aquelas relacionadas às questões culturais dos usuários; 
- Propor atividades durante o design que permitam a colaboração entre os participantes;

- Propiciar a criação de comunidades online para o design do REA.

O metamodelo de Santana (2019) é representado de maneira circular, mostrando que as dimensões são cíclicas e a dimensão produção está no centro, como um eixo que mantém as outras dimensões.

\section{Artefato de Arimoto et al (2016)}

Arimoto et al (2016) ressaltam que as iniciativas para promover o design e a criação de REA de qualidade com tempo e custos reduzidos ainda são incipientes, os autores trazem como contribuição da pesquisa um método ágil de desenvolvimento. O método, denominado AMOER, é baseado nas práticas de engenharia de software, com foco no planejamento e gerenciamento de projetos, e nas práticas de desenvolvimento de software. Ambos se concentram em aspectos colaborativos e flexíveis de desenvolvimento e melhoria de qualidade e produtividade, que são preocupações com relação ao desenvolvimento de REA.

Os autores desenvolvem o método com base em métodos ágeis, como SCRUM e XP, métodos que se concentram nos processos colaborativos e flexíveis de desenvolvimento, com foco na melhoria de qualidade e produtividade. O SCRUM é uma metodologia ágil que divide um projeto em pequenos objetivos, e distribui esses objetivos em sprints, que são ciclos curtos de desenvolvimento, nos sprints se estabelecem as atividades de cada membro de um time e as datas de fechamento. No SCRUM a comunicação é um fator importante para o ciclo de desenvolvimento, tanto durante quanto ao final de cada Sprint.

No modelo AM-OER a primeira etapa parte do levantamento das necessidades de aprendizagem, ou problemas identificados por educadores, para na sequência ser realizado um esboço de um REA que atenda essas necessidades. A equipe se reúne para planejar os sprints (e seu tempo de duração) e os objetivos dentro de cada sprint. Atribui-se também as funções dentro da equipe e as responsabilidades de cada objetivo. O REA é desenvolvido de forma iterativa e incremental através de sprints curtos, com reuniões entre os membros da equipe para verificar o progresso, receber feedback e melhorias. No SCRUM preza-se pela comunicação das dificuldades enfrentadas pelos integrantes de equipes, assim como sentimentos e emoções, então nessas reuniões é importante identificar obstáculos que dificultam o progresso do trabalho.

No final do Sprint a equipe se reúne novamente para fechamento das atividades do Sprint, e os autores propõem no modelo que partes de REA já aprovados por educadores sejam entregues aos alunos para feedbacks sobre como usar experiências de aprendizagem. Com os constantes feedbacks e em momentos frequentes do projeto, o ciclo de desenvolvimento se torna mais assertivo, com maior qualidade e um tempo mais otimizado. Esse ciclo iterativo acontece até que o REA possa ser entregue (Arimoto et al, 2016).

O que se evidencia na análise dos artefatos existentes é que os 8 artefatos selecionados reforçam a importância da colaboração para a educação aberta (e aprendizagem aberta) e a colaboração como ponto fundamental para o ciclo de vida REA. Destes, 6 artefatos propõem o 
uso de ferramentas de autoria e cocriação para incentivar a colaboração entre as partes envolvidas em um projeto REA, algumas ferramentas foram desenvolvidas especificamente para esse propósito, como é o caso do artefato de Leffa (2017).

O estudo descrito por Kim (2018) teve por objetivo propor um framework para integrar o uso de recursos educacionais abertos nas atividades de design de materiais educacionais pelos professores, o framework é composto por seis etapas e incentiva o trabalho colaborativo. Teodoroski (2018) desenvolveu um Ecossistema de REA no Brasil, com elementos para o processo de construção de projetos, considerando a sua complexidade, interdependência e dinamismo. $O$ ecossistema inicia com 8 elementos principais que se ramificam em mais elementos, o elemento "Produção" se desdobra ressaltando a colaboração, cocriação e coletividade. Nascimbeni e Burgos (2016) estabeleceram uma estrutura que apresenta caminhos de desenvolvimento para professores do Ensino Superior em quatro dimensões: design de aprendizagem, ensino recursos, estratégias de ensino e métodos de avaliação. Avila et al (2016) elaboraram um modelo denominado CO-CREARIA, que se trata de um modelo colaborativo para a criação e avaliação de recursos educacionais abertos baseados na metodologia ADDIE do design instrucional, o modelo contempla a co-criação e colaboração no sentido de contribuição e participação dos usuários na elaboração dos recursos. Nestes quatro artefatos citados, os autores reforçam a importância da construção colaborativa, porém não detalham como isso pode ser feito.

Dois artefatos foram detalhados neste artigo, pois apontam estratégias e diretrizes para o desenvolvimento colaborativo de REA. Um deles é o metamodelo de Santana (2019) que indica a colaboração como uma das dimensões para o design de REA. Na dimensão de colaboração são apontadas diretrizes para efetivação de atividades que buscam estruturar, regular e incentivar a participação no processo de design, e também o trabalho colaborativo entre os participantes. Nas diretrizes apontadas existe um alinhamento com o referencial teórico sobre colaboração e design colaborativo e nos pilares fundamentais para se estruturar uma colaboração disciplinada. O segundo é o artefato de Arimoto et al (2016) que associa metodologias ágeis ao design de REA, e essas metodologias, como o SCRUM, prezam por uma estrutura colaborativa, de trocas e organização de tarefas, e de comunicação constantes entre as partes, que também se alinham com o referencial teórico sobre colaboração e design colaborativo.

\section{Considerações Finais}

O presente artigo teve por intuito apresentar um estudo que objetivou analisar os artefatos existentes para auxílio ao desenvolvimento de Recursos Educacionais Abertos (REA), e identificar se e como a colaboração é abordada nos mesmos.

O estudo evidenciou que os artefatos analisados reforçam a importância da colaboração para a educação aberta (e aprendizagem aberta) e como ponto fundamental para o ciclo de vida REA. Outro fator enfatizado pelos recursos é a necessidade de construção colaborativa 
dos recursos. Os artefatos de Santana (2019) e Arimoto et al (2016) indicam diretrizes para que a colaboração possa ser integrada de maneira intencional e planejada. Associam com outras metodologias, como a do Design instrucional e com SCRUM. No SCRUM, utilizado por Arimoto et al (2016) a colaboração fica mais evidente e processual, porém ainda é necessário aprofundar como os participantes de um projeto podem conduzir as etapas de um REA de maneira colaborativa. Portanto, o estudo sugere que há uma importante demanda pelo design colaborativo de REA.

\section{Referências}

Arimoto, M. M., Barroca, L., \& Barbosa, E. F. (2016). AM-OER: An agile method for the development of open educational resources. Informatics in Education, 15(2), 205-233. https://doi.org/10.15388/infedu.2016.11

Avila, C., Baldiris, S., Fabregat, R., \& Graf, S. (2016). Cocreation and Evaluation of Inclusive and Accessible Open Educational Resources: A Mapping Toward the IMS Caliper. Revista Iberoamericana de Tecnologias Del Aprendizaje, 11(3), 167-176. https://doi.org/10.1109/RITA.2016.2589578

Conforto, E. C., Amaral, D. C., \& da Silva, S. L. (2011). Roteiro para Revisão Bibliográfica Sistemática: Aplicação no Desenvolvimento de Produtos e Gerenciamento de Projetos. Porto Alegre. Disponível em https://www.researchgate.net/publication/267380020_Roteiro_para_Revisao_Bibliografica_S istematica_Aplicacao_no_Desenvolvimto.pdf

Gil, A. C. (2008). Métodos e técnicas de pesquisa social. São Paulo: Editora Atlas.

Hansen, M. T. (2010). Colaboração: o segredo dos grandes líderes para evitar armadilhas, promover a união e conseguir excelentes resultados. Rio de Janeiro: Elsevier.

Hargrove, R. (1998). Colaboração criativa: a interação de talento e diversidade para obter resultados positivos. São Paulo: Editora Cultrix.

Kim, D. (2018). A framework for implementing OER-based lesson design activities for preservice teachers. International Review of Research in Open and Distance Learning, 19(4), 148-170. https://doi.org/10.19173/irrodl.v19i4.3394

Leffa, V. J. (2016). Uma Outra Aprendizagem É Possível: Colaboração Em Massa, Recursos Educacionais Abertos E Ensino De Línguas. Trabalhos Em Linguística Aplicada, 55(2), 353378. https://doi.org/10.1590/010318134942176081

Mallmann, E. M., \& Nobre, A. (2015). Dos Objetos De Aprendizagem Aos Recursos Educacionais (Abertos). Challenges 2015: Meio Século de TIC Na Educação, Half a Century of ICT in Education.

Marconi, M.; Lakatos, E.(2003). Fundamentos de metodologia científica. São Paulo: Editora Atlas.

Nascimbeni, F., \& Burgos, D. (2016). In search for the open educator: Proposal of a definition and a framework to increase openness adoption among university educators. International Review of Research in Open and Distance Learning, 17(6), 1-17. 
Okada, A. (2013). Recursos Educacionais Abertos \& Redes Sociais. Disponível em https://www.ces.uc.pt/myces/UserFiles/livros/1097_REA_REcursosEducAbertos.pdf

Pazmino, A. V. (2015). Como se cria: 40 métodos para design de produtos. São Paulo: Blucher.

Pirkkalainen, H.; Pawlowski, J. M.; Pappa, D. (2017). Educators' open educational collaboration online: The dilemma of emotional ownership. Computers and Education, v. 106, n. December, p. 119-136.

Santana, E. C. (2019). Um Metamodelo Para O Processo De Design De Recursos Educacionais Abertos Multiculturais. [Tese de doutorado] Programa de Pós-graduação em Engenharia Elétrica e Computação. Universidade Presbiteriana Mackenzie, São Paulo, SP, Brasil.

Teodoroski, R. D. C. C. (2018). REA no Brasil: construção de um modelo ecossistema de REA. [Tese de doutorado] Programa de Pós-graduação em Engenharia e Gestão do Conhecimento. Universidade Federal de Santa Catarina, Florianópolis, SC, Brasil.

Zancanaro, A. (2015). Produção De Recursos Educacionais Abertos Com Foco Na Disseminação Do Conhecimento: Uma Proposta De Framework. [Tese de doutorado] Programa de Pós-Graduação em Engenharia e Gestão do Conhecimento. Universidade Federal de Santa Catarina, Florianópolis, SC, Brasil.

\section{Sobre o(a/s) autor(a/es)}

Gabrielle da Fonseca Hartmann Grimm, Ma., UFPR, Brasil <gabihgrimm@gmail.com> Adriano Heemann, Dr., UFPR, Brasil <adriano.heemann@ufpr.br> 